\title{
Enfermedad pulmonar obstructiva crónica (EPOC): visión global y continuidad de cuidados
}

Escarrabill J. Enfermedad pulmonar obstructiva crónica (EPOC): visión global y continuidad de cuidados. An Med Interna (Madrid) 2003; 20: $337-339$.

La enfermedad pulmonar obstructiva crónica (EPOC) es la cuarta causa de muerte. La carga asistencial que representa esta enfermedad aumenta progresivamente, tanto en el número de ingresos hospitalarios como en las visitas en el Servicio de Urgencias, especialmente en los grupos socioeconómicos menos favorecidos (1). A pesar de la importancia de la enfermedad y del impacto de la misma sobre el sistema sanitario (2), una parte significativa de los pacientes con EPOC no se diagnostican (3) o bien el tratamiento se realiza equiparándolo con el del asma, pero sin el mismo entusiasmo. Esta situación la ha resumido Robin Taylor (4) hablando del nihilismo negligente al hacer referencia al tratamiento de la EPOC.

En los últimos cincuenta años la confusión terminológica ha favorecido poco el conocimiento de la enfermedad y el abordaje práctico de la misma. Durante años han coexistido, en muchas ocasiones como sinónimos, enfisema, bronquitis crónica, bronquitis asmática, enfermedad de pequeñas vías o bronquitis crónica simple u obstructiva. A principios de los 80, veinticinco años después del simposium Ciba (1959), se constaba el escaso éxito del término enfermedad obstructiva crónica por la competencia del acrónico norteamericado COPD (chronic obstructive pulmonary disease) (5). A pesar de todo, COPD (o EPOC en español) se ha introducido progresivamente en todos los ámbitos haciendo émfasis en la relación de la enfermedad con el tabaco (en los paises occidentales), en la obstrucción poco reversible (o por lo menos no tan reversible como en el caso del asma) y en el papel básico de la espirometría (6) en el diagnóstico de la misma.

La historia natural de la EPOC es variable. Aunque únicamente la deshabituación tabáquica y la oxigenoterapia domiciliaria cambia positivamente la evolución, existen tratamientos que mejoran los síntomas y, posiblemente, la calidad de vida. Es bien conocido que no todos los fumadores desarrollan obstrucción al flujo aéreo e identificar a los fumadores "susceptibles" es uno de los grandes retos para los investigadores. Se han descrito fenotipos distintos en pacientes con EPOC con manifestaciones clínicas similares. La identificación de los fenotipos específicos relacionados con la EPOC puede mejorar el diagnóstico y el tratamiento (7). La heterogeniedad y el poco conocimiento de la EPOC podría explicarse al ser una enfer- medad genotípicamente compleja que evoluciona lentamente. Así, desde un punto de vista teórico, la identificación de diversos fenotipos podría explicar la existencia de pacientes con EPOC en los que predomina la destrucción (enfisema), la pérdida de elasticidad o la hiperreactividad bronquial, o bien, el caso de pacientes con obstrucción en edades relativamente jóvenes o con un declive acelerado de la función pulmonar. Los diferentes fenotipos de la EPOC quizás tambièn podrían explicar las manifestaciones sistémicas (8), como la respuesta inflamatoria sistémica, los alteraciones nutricionales, la disfunción del músculo esquelético o los efectos potenciales cardiovasculares o sobre el metabolismo óseo (9).

En estos momentos la introducción de nuevos fármacos, los resultados del estudio del National Emphysema Treatment Trial Research Group (10) sobre la indicación de la cirugía de reducción de volumen, el trasplante pulmonar o la rehabilitación respiratoria (11), o las estrategias para tratar la dependencia al tabaco (12) abren expectativas para mejorar el tratamiento a largo plazo de los pacientes con EPOC (13).

Sin embargo, a pesar del papel evidente de las novedades técnicas o farmacológicas no se debería olvidar que la clave está en dos estrategias todavía poco desarrolladas en la práctica asistencial cotidiana: el papel del paciente en la toma de decisiones y en sus expectativas respecto a la atención y, como consecuencia o en relaciación con lo anterior, la necesidad de organizar la atención de los pacientes con EPOC de una manera muy distinta a la actual.

Las expectativas de los pacientes con EPOC respecto a las características del tratamiento que desean recibir podrían resumirse en tres dimensiones de tiempo, espacio y contenido, como en la mayoría de procesos crónicos. Los pacientes esperan recibir el tratamiento idóneo sin tiempos de espera inexplicables, con accesibilidad a todos los dispositivos asistenciales y con un contenido completo (atención integral), de manera que no deban acudir por su cuenta a distintos proveedores para recibir lo que necesitan (14). Hay evidencias que demuestran que la implicación de los pacientes ha provocado cambios en la provisión de servicios sanitarios (15) o en la toma decisiones. La decisión final no se ve influida únicamente por la evidencia si no que, además de la evidencia disponi- 
ble, las circunstancias (perspectivas y habilidades del médico) y los valores del paciente juegan un papel determinante (16). También hay que considerar el hecho de que el paciente, una vez recibida la información, no se implique en la toma decisiones, delegando implícitamente en el médico (17). Se requieren más estudios para fundamentar el impacto real de estas intervenciones.

Los programas educativos para promover el auto-cuidado se han dirigido a la deshabituación tabáquica, mejorar ele ejercicio, nutrición, auto-tratamiento de las agudizaciones, técnicas de inhalación o adaptación a las actividades de la vida diaria. Cuando se analiza el papel del auto-cuidado (selfmanagement) en la evolución de la EPOC no se han encontrado evidencias concluyentes del impacto de estas estrategias (18). Es posible que la adquisición de habilidades y conocimientos no implique directamente un cambio de conducta o, quizás, dado que las intervenciones en la EPOC son multifactoriales sea difícil individualizar los efectos de uno de los componentes. Así, en el impacto de la hospitalización a domicilio, la educación del paciente juega un papel muy importante, además de otros aspectos de la intervención, como la visita domiciliaria o la accesibilidad al equipo (19). Hay trabajos que sugieren que la educación para promover el auto-cuidado reduce la medicación de rescate durante las agudizaciones de la EPOC (20) o el uso de recursos sanitarios (21).

La mayor parte de los dispositivos asistenciales están organizados para tratar procesos agudos. La educación de los profesionales sanitarios, en líneas generales, se diseña para dar respuesta a este tipo de organización. Por lo tanto, en estos momentos el reto sanitario más importante es dar respuesta a las necesidades de pacientes con enfermedades crónicas. Este problema se plantea en las sociedades occidentales, en las que las causas no infecciosas de morbimortalidad son las más importantes. Pero también en los paises en vías de desarrollo se plantea el problema de la atención a los pacientes con enfermedades crónicas que, en este caso, coexiste con los problemas relacionados con las enfermedades infectocontagiosas.

Cuando los dispositivos asistenciales diseñados para tratar procesos agudos deben dar respuesta a las necesidades de los pacientes con enfermedades crónicas surgen problemas. Estos dispositivos suelen dar respuestas fragmentadas, y a menudo duplicadas ante la ineficiencia de la transmisión de información. Además no satisfacen las necedidades globales de los pacientes. La atención a pacientes con enfermedades crónicas como la EPOC debe tener en cuenta la presencia de comorbi- lidades y de condiciones asociadas (problemas sociales o trastornos cognitivos) (22). La atención integral (capaz de dar respuesta a todas las necesidades del paciente) y la continuidad asistencial son cruciales.

La respuesta de los sistemas sanitarios no puede basarse en soluciones simples y unidimensionales. La solución a la atención adecuada de los procesos crónicos no pasa solamente por el acceso a la medicación o a procedimientos terapéuticos específicos. Es preciso invertir en un nuevo modelo asistencial adaptado a las necesidades de los pacientes con enfermedades crónicas. Los equipos multidisciplinarios constituyen la base del tratamiento de los pacientes con enfermedades crónicas (23). Un equipo multidisciplinario incluye a todos los profesionales que intervienen significativamente en la toma de decisiones y en la realización del tratamiento. Estos profesionales se comunican regularmente y toman decisiones sobre un grupo concreto de pacientes. El trabajo en equipo implica la transferencia de responsabilidades, especialmente del médico a otros profesionales, y la consolidación de figuras que intervienen activamente en la organización y la coordinación de los cuidados (como es el caso de la figura conocida como enfermera/o responsable de la gestión de casos).

La Organización Mundial de la Salud identifca seis áreas de mejora en el tratamiento de las enfermedades crónicas (24): a) Mejorar la cooperación entre dispositivos asistenciales, b) Organización de los cuidados, c) Herramientas educativas para promover el autocuidado, d) Cambios en la oferta de servicios (importancia de la participación de otros profesionales), e) Herramientas de soporte a las decisiones (protocolos o guías de práctica clínca) y f) Sistemas de información que permitan compartir información on-line.

Los avances en el tratamiento de los pacientes con EPOC deben mucho a la introducción de innovaciones técnicas o a los nuevos fármacos. Pero el progreso será limitado sin un cambio en la organización de la atención en el que la visión global de las necesidades del paciente y la continuidad asistencial sean los pilares básicos.

\section{J. ESCARRABILL}

UFISS-Respiratòria (Servei de Pneumologia). Hospital Universitari de Bellvitge. L'Hospitalet de Llobregat. Barcelona

\section{Bibliografía}

1. Price D, Duerden M. Chronic obstructive pulmonary disease. BMJ 2003; 326: 1046-7.

2. Pauwels RA, Buist AS, Calverley PM, Jenkins CR, Hurd SS and the GOLD scientific committee. Global strategy for the diagnosis, management, and prevention of chronic obstructive pulomary disease. NHLBI/WHO Global obstructive lung disease (GOLD) workshop summary. Am J Respir Crit Care Med 2001; 163: 1256-76. (www.coldcopd.org).

3. Rennard S, Decramer M, Calverley PMA, et al. Impact of COPD in North America and Europe in 2000: subjects perspective of Confronting COPD International Survey. Eur Respir J 2002; 20: 799-805.
4. Robin Taylor D. Chronic obstructive pulmonary disease. BMJ 1998; 316: 1475.

5. Fletcher CM, Pride NB. Definitions of emphysema, chronic bronchitis, asthma, and airflow obstruction: 25 years on from the Ciba symposium. Thorax 1984; 39: 81-5.

6. A report to the Medical Research Council by their committee on the aetiology of chronic bronchitis. Definition and classification of chronic bronchitis for clinical and epidemiological purposes. Lancet 1965; i: 775-9.

7. Boschetto P, Miniati M, Miotto D, Braccioni F, De Rosa E, Bononi I, et al. Predominant emphysema phenotype in chronic obstructive pulmo- 
nary. Eur Respir J 2003; 21: 450-4.

8. Barnes PJ. Chronic obstructive pulmonary disease. N Engl J Med 2000; 343: $269-80$

9. Agustí AGN, Noguera A, Sauleda J, Sala E, Pons J, Busquets X. Systemic effects of chronic obstructive pulmonary disease. Eur Respir J 2003; 21: 347-60.

10. Ramsey SD, Berry K, Etzioni R, Kaplan RM, Sullivan SD, Wood DE. National Emphysema Treatment Trial Research Group. N Engl J Med. 2003; 348: 2092-102.

11. Cost effectiveness of lung-volume-reduction surgery for patients with severe emphysema.

12. Lacasse Y, Wrong E, Guyatt GH, King D, Cook DJ, Goldstein RS Meta-analysis of respiratory rehabilitation in chronic obstructive pulmonary disease. Lancet 1996; 348: 1115-9.

13. Anderson JE, Jorenby DE, Scott WJ, Fiore MC. Treating Tobacco Use and Dependence. Chest 2002; 121: 932-41.

14. Celli B. EPOC: desde el nihilismo no justificado a un optimismo razonable. Arch Bronconeumol 2002; 38: 585-8.

15. Bridging the gap. Commissioning and delivering high quality integrated respiratory healthcare. A report from the Respiratory Alliance. London, 2003.

16. Crawford MJ, Rutter D, Manley C, Weaver T, Bhui K, Fulop N, et al. Systematic review of involving patients in the planning and development of health care. BMJ 2002; 325: 1263-7.

17. Haynes RB, Devereaux PJ, Guyatt GH. Physicians' and patients' choices in evidence based practice. BMJ 2002; 324: 1350.
18. Perpiñá M, Sobradillo V, Castillo J, Duce F, et al. Búsqueda de información y toma de decisiones en pacientes asmáticos. Arch Bronconeumol 1999; 35: 435-439.

19. Monninkhof EM, van der Valk PD, van der Palen J, van Herwaarden CL, Partidge MR, Walters EH, et al. Self-management education for chronic obstructive pulmonary disease. Cochrane Database Syst Rev 2003; (1): CD002990.

20. Hernandez C, Casas A, Escarrabill J, Alonso J, et al. Home hospitalization of exacerbated copd patients. A randomized controlled trial for clinical efficacy and cost analysis of innovative services for chronically ill patients. Eur Respir J 2003; 21: 58-6.

21. Gallefoss F, Bakke PS. Cost-benefit and cost-effectiveness analysis of self-management in patients with COPD-- a 1-year follow-up randomized, controlled trial. Respir Med 2002; 96: 424-31.

22. Bourbeau J, Julien M, Maltais F, Rouleau M, Beaupre A, Begin R, et al. Chronic Obstructive Pulmonary Disease axis of the Respiratory Network Fonds de la Recherche en Sante du Quebec. Reduction of hospital utilization in patients with chronic obstructive pulmonary disease: a disease-specific self-management intervention. Arch Intern Med. 2003; 163: 585-91.

23. DeBusk RF, West JA, Houston Miller N, Taylor CB. Chronic Disease Management. Arch Intern Med 1999; 159; 2739-42.

24. Wagner EH. The role of patient care teams in chronic disease management. BMJ 2000; 320: 569-71.

25. World Health Organization. Innovative Care for Chronic Conditions. Meeting Report. 2001. 